\title{
Application of Web-based Gis in Municipal Territory Registration Management
}

\author{
Genti Qirjazi ${ }^{1}$, Bujar Drishti ${ }^{2}$ \\ ${ }^{1}$ Department of Geodesy, Polytechnic University of Tirana, Albania \\ ${ }^{2}$ Department of Geodesy, Polytechnic University of Tirana, Albania
}

\begin{abstract}
GIS technology has enabled the possibility to analyze and define decision making by managing geospatial information. With the rapid development of the technology, GIS tools and analysis can be operated through internet communications. GIS as web services enables us to implement tools and services which are accessible trough the web and can be used and managed by every user. Web GIS technologies has enabled the possibility to manage and analyze geospatial information through web services. In this article we are showing a web-based GIS solution for managing local government property taxes through analyzing different geospatial data. The GIS application will facilitate property tax collection and administration through creation of the tax-related elements of a working territory register. The web-based application's primary functionality is to support parcel- and building-based query, selection, display, and mapping.
\end{abstract}

Keywords: GIS, Geoserver, GIS Services, WEBGIS

\section{Introduction}

GIS technology has enabled the possibility to analyze and define decision making by managing geospatial information. With the rapid development of the technology, GIS tools and analysis can be operated through internet communications. GIS as web services enables us to implement tools and services which are accessible through the web and can be used and managed by every user. Web GIS technologies has enabled the possibility to manage and analyze geospatial information through web services. In this article we are showing a web-based GIS solution for managing local government property taxes through analyzing different geospatial data. The GIS application will facilitate property tax collection and administration through creation of the tax-related elements of a working territory register. The web-based application's primary functionality is to support parcel- and building-based query, selection, display, and mapping. We have utilized best available spatial data layers about the municipality's roads and buildings from base map imagery. Data on roads and buildings are utilized to generate a numbered address system for the municipality.

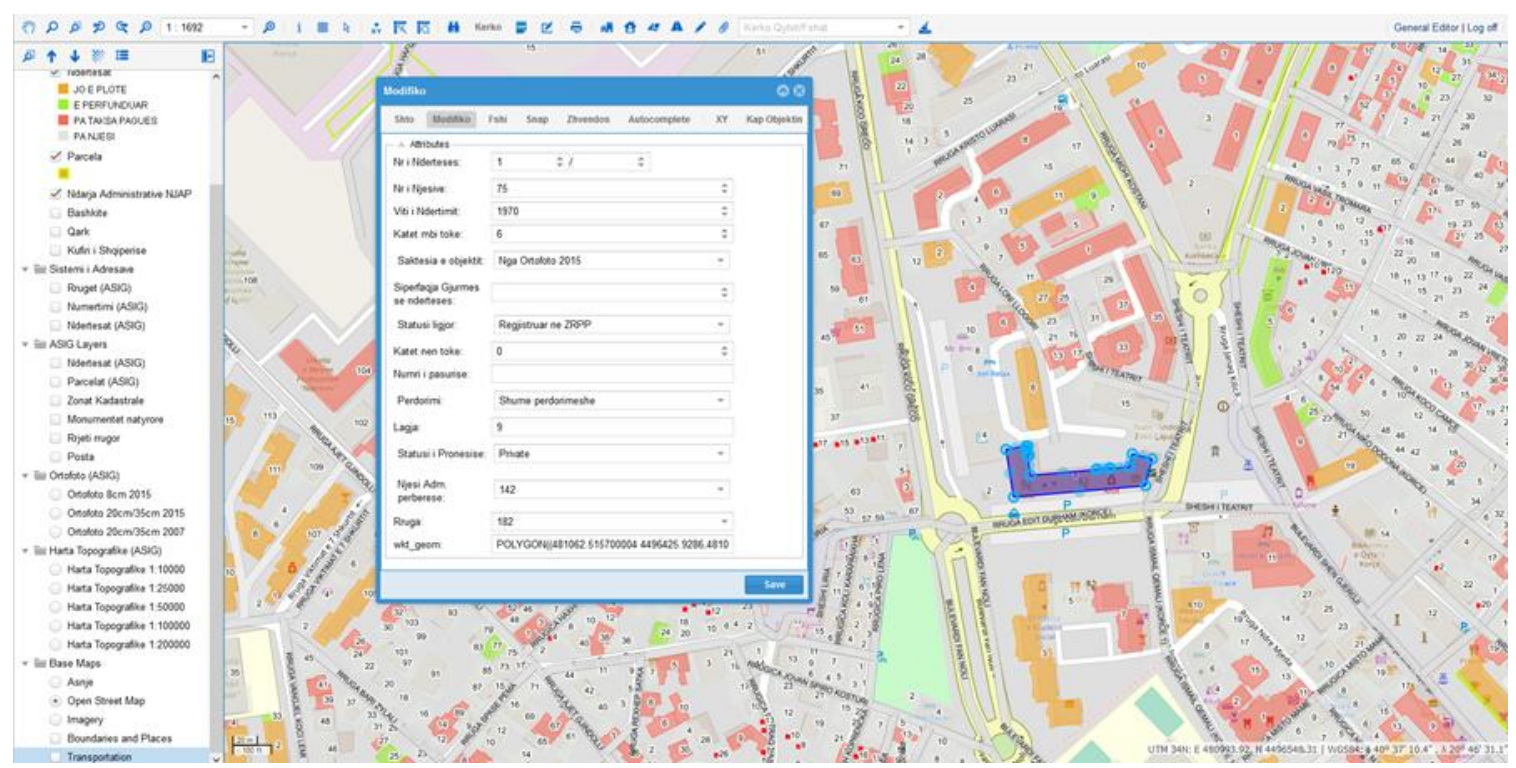

Figure 1: User interface of the WebGIS Application

We have created a relational data structure to link building to property, property to owner, property to water utility customer number (where applicable), owner to civil registry information, and address to building and property. Territory register fields will thus include building, cadastral, parcel, and ownership information. Data tables will be populated by existing data and new data collected in the field and recorded in the geodatabase by municipal staff.

General description of Web-Based GIS Application

WebGIS will relate the TAIS (Tax Administration Information System) database records with the geographic 
location. This will enhance the existing application with the functionality of locating geographically each taxpayer's information.

Adding the geographic dimension to the TAIS application will ensure several benefits such as:

- Possibility to check if the tax amount is appropriate related with the ownership location;

- Identification of the tax amount based on the inhabited zone category;

- Possibility to design a division of the inhabited area in tax zones and calculate automatically which are the ownerships that fall in a certain zone;

- Identification in the map of all the buildings (with the related apartments) and ownerships which are not registered in the tax system;

- Full control over the existing and the new ownerships, because it is possible to identify in map a possible ownership not included in the TAIS application;

- It accomplishes the TAIS application with better planning of the taxes by getting more complete analysis using geographic component;

- It gives the possibility to visualize geographically any attribute of the tax database, such as where are located people that are not paying taxes, which area is paying a bigger amount of taxes compared with the rest of the inhabited area, where there are frequent delays in tax payments, etc. These geographic visualization of the attributes helps the officials to take better decisions;

- Gives the possibility divide geographically the areas of each inspector and then display graphically the results of the work for each inspector, identify the necessity of redefining the zones, identify geographically where are located the major problems and take better measures to solve these;

- Using the geographic dimension, a new world of analysis based on spatial location is open, such as, find the properties which are in a certain distance from a road, find the properties which fall in a specified category of regulatory plan, etc.

The Web GIS application is accessible through using at least one current web browser such as Mozilla Firefox, Google Chrome, Internet Explorer, etc. resulting in: (a) no need to install any additional software in user computers; (b) no need for powerful user computer, as it will only need enough power to be able to use a web browser; and (c) only an internet connection will be required. The application satisfies all OGC (Open Geospatial Consortium) standards, ensuring in this way sharing of information. All software required to build the WebGIS application will use OpenSource platforms (free of charge), so there will be no additional costs in buying licenses for commercial software.

The WebGIS application can display Satellite Images (in background) such services from Google, Bing or/and map services such as Google, Bing, OpenStreet. This will enable the users to have the view of the area as near as possible to the reality and digitizing geographic features ex. such as missing buildings and the new ones. The application will also display other raster data sources, such as orthophotos, topographic maps, etc. through existing public WMS. The user can choose which of the available sources is going to be used as background. In Albania there is created recently ASIG (State Authority for Geospatial Information), which provides all the above-mentioned information in the form of free WMS/WMTS services.

The WebGIS application will display all available information organized in layers. The user will have the possibility to make these layers on/off and create the required map view by itself. The list of layers can include buildings, cadastral parcels with property number, roads/streets, addresses (ex. from ASIG) etc. The property number together with the number of the cadastral zone will be the key information to link the geographical objects with the TAIS database records.

The user will be enabled to navigate the map with all required tools (as zoom in/out, pan, etc.). The application will provide tools for coordinates, distance, and area measurements, dynamic scale and coordinates display, information retrieve for any of the drawn layers, etc.

The main functionality of the WebGIS application will be the possibility to add/edit ownership/building footprints together with the possibility to link any property object with the taxpayer information from TAIS software. This will be provided through an easy and user-friendly interface. The application will provide tools to ensure the geometry correctness of the drawn objects. The add/edit functionalities will be provided to registered users through definitions of roles using a dedicated Administration Panel. The editing functionalities will not only limit to these data layers but will function too in each other editable layer such as roads/streets, address attributes, etc.

The Web GIS application will also provide attribute and spatial queries (depending on the available additional layers). For instance, the users can search and query using building/apartment number, tax payer name, etc. as well as using relationships between geometrical objects, such as finding all buildings that are inside a user specified area, giving a list with all related apartments and all the attribute data.

Any list of objects generated from query/selection functions will have the possibility to export as table with all the related object attributes in a spreadsheet format manageable from Excel.

It will be possible to identify the apartment records which will not have correspondence between geometry representation and tax database records. This will ensure that no building in the WebGIS application is leaved with no data from TAIS application and vice versa.

\section{Software Platform area}

- Server Operating System will be Windows Server 2008 R2 or higher

- The data can be stored and managed on a Relational Database Management System. PostgreSQL is a powerful, open-source object-relational database system and it is going to be used for this purpose. PostGIS is a project 
which adds support for geographic objects in PostgreSQL, allowing it to be used as a spatial database for geographic information systems (GIS), much like ESRI's SDE or Oracle's Spatial extension. The combination of these two software will enable the store of attribute and spatial data (geographic) in the same dBase.

- GeoServer (open source software server) will be used you to display (publish) spatial information in the web. This will be done through Web Map Service (WMS), Web Feature Service (WFS) and Web Coverage Service (WCS) standards.

- OpenLayers will be used for the web client, it can display map data in web browsers. It is completely free, OpenSource JavaScript Library and also it is integrated into
GeoServer. Additional Open-Source JavaScript Libraries can be used for user interface development of the WebGIS application.

- IIS will be used as web server.

The application will have two main components: the server and the web-client(s). On the server side will be stored processed and analyzed the data in spatially enabled database. The client will be used to view and visually manage the data, as well as enter new data.

The Web-GIS application architecture are schematically presented in and Figure 2:

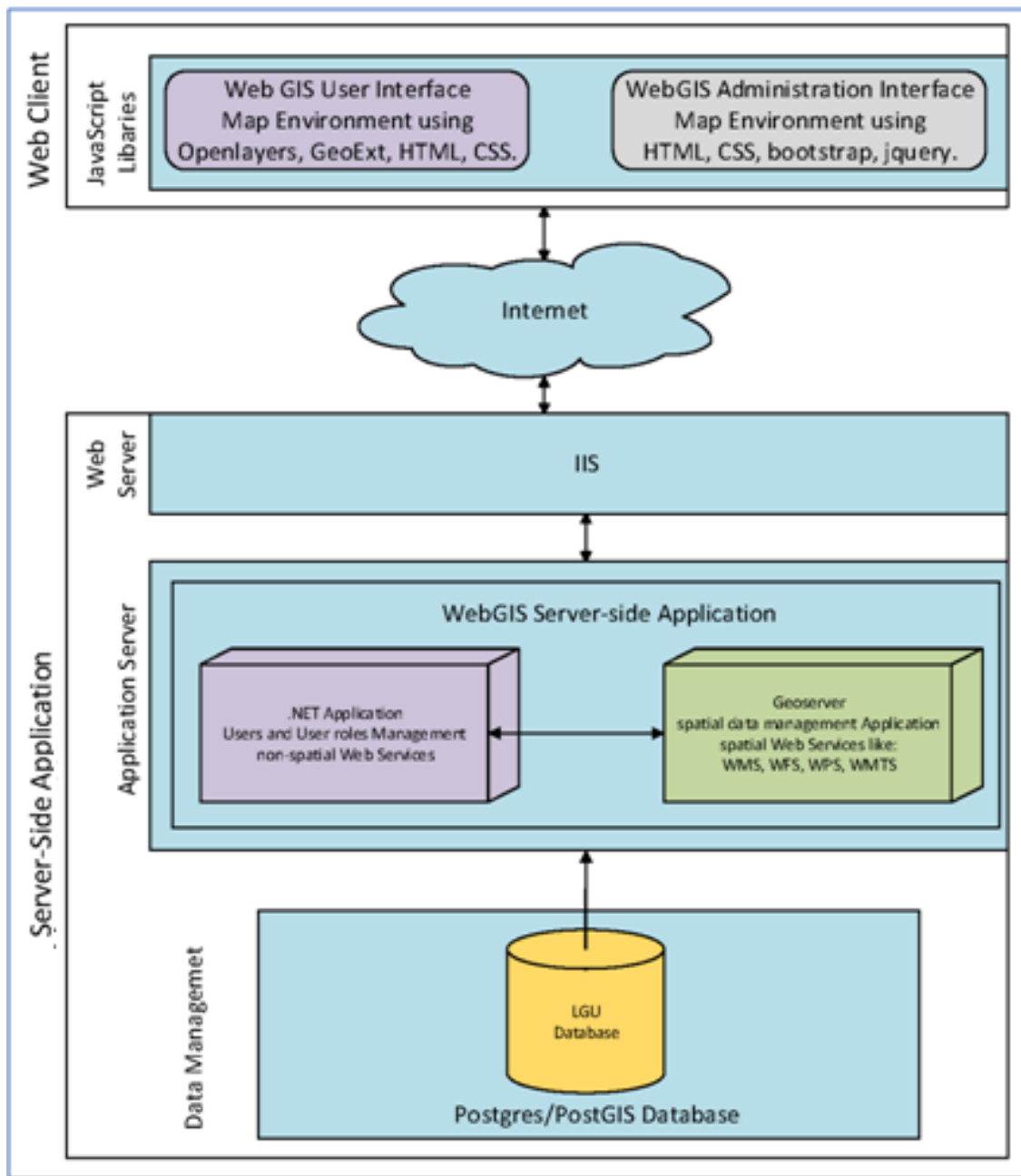

Figure 2: WebGIS Application Schema

The WebGIS application will link with the TAIS Central Database to link the geographic objects with the taxpayer information.

The Web-GIS Application will implement using the following hardware Infrastructure which is schematically presented in the Figure 3: 


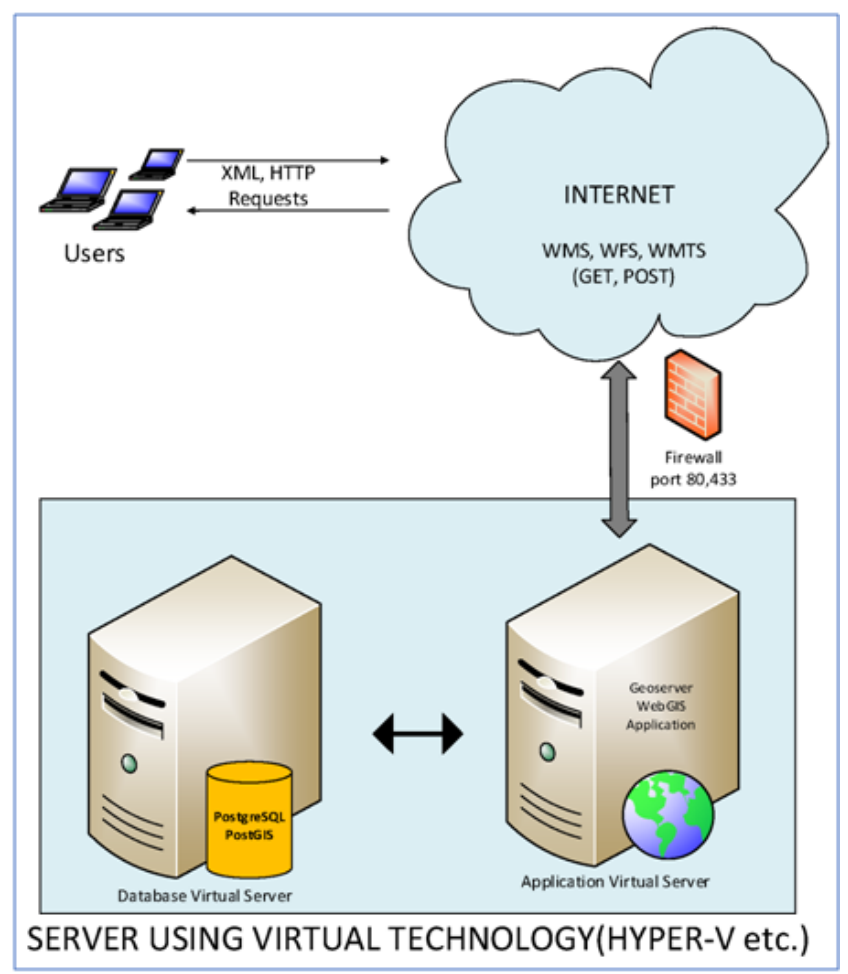

Figure 3: Hardware Architecture Schema

\section{Web-GIS Functionalities / Tools}

\section{General functionality}

1) Multi-user environment;

2) Accessible from popular browsers (Mozilla Firefox, Google Chrome, Internet Explorer, etc.);

3) The WebGIS application will be mobile friendly for usage in mobile web browsers. It will have the possibility to read the mobile location coordinates and draw the locations in the specific vector layer of the application together with some attributes;

4) Spatial data will be organized in layers. The Web-GIS Application will have a set of so called "Base Layers". For instance, one of these can be Google Satellite Images. But also there will be the possibility to add more than one base layer, that can be any satellite or/and aerial photography available for the country, topographic maps, Bing Satellite Image, etc. (ASIG already delivers WMS for many layers and between them orthophoto, topographic maps, etc.). WebGIS will have the functionality to display as Base Layer following information:

- Google and Bing (map and image), OpenStreet Map;

- Orthophoto layer from ASIG;

- Ability to view and deliver layers of information through use of WMS/WMTS services;

- Ability to allow the user to manually add a not predefined WMS/WMTS service for use in a WebGIS session.

The user can select which of them to show in background of the other data. When the local data are not available the Web-GIS App can always have Google Satellite, Bing Satellite Image, or other similar web services.
The second set of layers will consist of data in vector format saved in the WebGIS application database. All these layers will be editable. Based on the existing digital information from the RFP document, following is the list of the vector layers WebGIS Application will manage:

- Building layer (normally polygon geometry representing the buildings footprint). Each building will link with its property number. It will be possible to add apartment numbers for each building and also link these apartments with the owners registered in the tax application database (when a correct cadastral zone and property number is registered in the database). Taking in consideration that the information regarding the property number by IPRO is missing in many cases, the WebGIS application will assign to each property a unique identification number. This ID could be used to link the appropriate taxpayer information in the TAIS database. Also the WebGIS application will have the possibility to register for each property the owner/owners information and display this information in a spreadsheet compatible format for the selected objects.

- Urban and agriculture land parcels (polygon geometry);

- Addressing layer with point geometry which will show the building entrances.

- Taxpayer information geographic layer. This layer will contain geographic information related with ownership layers (buildings/apartments and parcels). The layers will link with the taxpayer database attributes and will enable the presentation on the map of these attributes, such as different coloring/symbols to show the tax payment status (where are problems in tax payment), or show in map where are the ownerships not linked with tax payment database, etc. The user will have the possibility to select the category of tax payment information he is interested in a specific moment;

- Management zones (polygon geometry);

- Tax information layer such as property values zones (polygon geometry);

- Roads/streets with names (line geometry);

- Administrative division (polygon geometry);

- Reporting layer (not visible to any WebGIS user) manageable from GIS System coordinator for registering user reported. This layer will contain specific locations in the map where something needs to be reported, such as information for e new building, information on changes in an existent building, information about a new road/street or changes in the existing ones, report about a not correct information, etc. this information will be filled by local users and the system administrator is informed about each case (together with the reasons of reporting) and will have the possibility to study the cases and take the required decisions.

- Field data collection layer, which will be used to register online field data from mobile web browsers. This layer will be used to register the real time point coordinates taken during field work of the experts. This information can be elaborated latter in the office for updating the vector layer information (a smartphone 
with location functions and with internet access will be required to use this feature);

- Other layers depending on the existing digital data.

The geographic vector layers will have appropriate attributes such as addressing, ownership identification (ex. cadastral zone and property number), water utility/electric power customer number (where applicable), etc. The quantity of the attributes to migrate in the WebGIS database will define by the existing digital data. But the application will have the possibility to fill latter these attributes. The final attribute field structure for vector layers will define after analyzing the existing data.

The user can make visible/invisible any layer from this set and also can combine the visibility of the layers to have the required representation of the map. The layer data can be shown in categories (using different colors/symbols) depending on the attribute data they have. The user will have the possibility to define the transparency for each WebGIS layer;

- The Web-GIS application will display dynamically the coordinates of the actual mouse cursor position (it is possible to give the coordinates both in geographic and projected coordinate system);

- The actual scale of the map will show in a window; the user will have the possibility to select (in this window) from a list of predefined scales for the map viewer. The scale of the map can show also graphically as scale bar;

- The application can have a north arrow symbol;

- Context sensitive help tool and e brief online user manual for WebGIS functions.

\section{Base functions}

- Tools for navigation in the map. These will include "Zoom In" and "Zoom Out" by clicking in the map or by drawing a rectangle around the area of interest. "Fixed Zoom In" and "Fixed Zoom Out" with no need to click on the map; zoom in/out will be done automatically by clicking the button keeping the center of the actual map view and zooming with a predefined factor. "Zoom Previous"/"Zoom Forward" to go back and forth in the map views. "Pan" to shift the map in the desired direction. "Zoom Extent" to bring in the screen all the layers extent. "Zoom to Layer" to zoom in the extent of the selected layer. "Zoom to Selection" to bring into the screen all the actually selected objects of the map. The application can have also a slide zoom tool;

- Tools for distance and area measurement. The user can measure the distances and areas and also can get the coordinates of the desired point;

- Information Tool. Get information for any object in actual visible layers of the map. Possibility to display attributes of the captured objects in different layers. Possibility to activate hover capability for attribute information;

- Possibility to select objects by clicking or drawing different area shapes and list the attributes of the selected objects;
- Print Tool (produce PDF file format). The user will have the possibility to print (produce PDF file format) a desired area of the map in a selectable scale and in selectable paper format. There will be the possibility to add a title and show the scale.

\section{Draw/Edit functions}

- Draw polygons, points, lines in any of editable data layers with the correct geometry of the layer data. The adding of the features (ex. buildings) can be done digitizing by following the Google Satellite Image or other images as more detailed orthophoto/satellite image or any other WebGIS Base Layer. There will be possible to digitize features (ex. buildings, etc.) also by entering the coordinates of the vertices. The coordinates of the features can be measured in the field using a GPS Unit. The editing of the features can be done also by importing into the WebGIS locally saved GIS format files (such as KML and SHP); the user can see the imported objects in the WebGIS, modify them as required and save these objects in the appropriate layer adding the required attributes. There will be another possibility to enter a big quantity of data for support WebGIS vector layers; the user can elaborate the dataset from the field measurement using a desktop GIS software (there are free ones as Quantum GIS, GvSIG, UDig, etc.) creating a whole set of buildings, save this in a common geographic data format (let say Google Kml, ESRI Shape, etc.). The WebGIS application will have the possibility to upload this directly in the database. We suggest to use this functionality for the tax zoning/management layers but not for property related layers;

- Edit features geometry in any editable data layer. Editing functions can be, move object, move vertex/point, delete, etc.;

- Feature snapping functionality (endpoint, vertex, point, etc.);

- Enter the required attribute for newly added features and edit the attributes for existing features in any editable data layer. Possibility of using predefined lists of values for the required attribute fields to facilitate and speed the data entry;

- Functions to prevent incorrect geometries to be saved in the data layer;

- Ability to attach documents (such as JPEG or other raster formats, PDF, DWG), video as well as textual attribute information to the objects of the editable vector layers. Possibility to manage the attached documents (delete, reattach, etc.).

\section{Attribute Query (and Select) functions}

- Select objects from the actual layer by clicking on them, possibility for multi selection. Display the table with the attributes of the selected objects;

- Search for location based on GPS Coordinates;

- Tool for attribute query for each available vector layer. The user can search the attribute table of the selected layer by any field using different operators (as =, >, <, LIKE etc.). Where is needed, the searched value can be selected from a list of existing values without having to type it (as Property Number, etc.). Logical operands AND/OR can be used to combine several attributes. The 
results of the query will be displayed on the map and as a list of selected records;

- Mail merge function with owner and address data for the selected property objects, possibility to export the attribute data in a spreadsheet format (manageable from Excel);

- Possibility to export a table with selected records attribute data in a spreadsheet format (manageable from Excel).

\section{Spatial Query (and Select) functions}

- Tools for selecting features from one layer using spatial relationships (as intersect, within, disjoint, buffer, etc.) with the features of another layer. Ex. Select all the buildings which fall inside a certain tax area;

- Mail merge function with owner and address data for the selected property objects, possibility to export the attribute data in a spreadsheet format (manageable from Excel);

- Possibility to export a table with selected records attribute data in a spreadsheet format (manageable from Excel).

WebGIS Administration Module

- Possibility to manage roles and users. Define user roles, create/modify users, manage user credentials, etc.;

- Management of list value for predefined fields;

- Management of user activity (who, when, what log activity).

Users and User Roles of WebGIS Application

The WebGIS application will have e set of predefined user roles and management through a dedicated Administration Module. In the table below are listed the roles and a user case description for each of them:

\begin{tabular}{|c|c|}
\hline Roles & escription \\
\hline Administrator & $\begin{array}{l}\text { The users that are part of this role have the possibility to create and manage users and assign roles to them, manage } \\
\text { attribute list data and manage user log activities. } \\
\text { - Will log in to the application with username and password; } \\
\text { - Administration panel will open after authentication; } \\
\text { - Will have the possibility to create users; } \\
\text { - Will have the possibility to assign roles to the created users; } \\
\text { - Will create/update attributes list for the WebGIS layers; } \\
\text { - Will have the possibility to check and manage WebGIS users log activity. }\end{array}$ \\
\hline $\begin{array}{l}\text { Spatial Data } \\
\text { Editor }\end{array}$ & $\begin{array}{l}\text { This role is assigned to the users by the administrator and gives them the possibility to add and modify the spatial data } \\
\text { layers such as: buildings, parcels, roads/streets, tax information layer, etc. } \\
\text { - Will log in to the application with username and password; } \\
\text { - He will be redirected to the map environment after authentication; } \\
\text { - Will have the possibility to use map navigation (zoom, pan), measure function (distance, area), get info, printing, search } \\
\text { and query; } \\
\text { - Will have the possibility to add/modify vector geographic layer objects. }\end{array}$ \\
\hline $\begin{array}{l}\text { Data Entry } \\
\text { Editor }\end{array}$ & $\begin{array}{l}\text { This role is assigned by the administrator and gives to the users the possibility of data entry for tax zones information, etc. } \\
\text { - Will log in to the application with username and password; } \\
\text { - He will be redirected to the map environment after authentication; } \\
\text { - Will have the possibility to use map navigation (zoom, pan), measure function (distance, area), get info, printing, search } \\
\text { and query; } \\
\text { - Will have the possibility to add/modify the information about tax zones, etc. }\end{array}$ \\
\hline $\begin{array}{l}\text { Field data } \\
\text { collector }\end{array}$ & $\begin{array}{l}\text { This role is assigned by the administrator and gives the users possibility of data entry in the Field data collection layer. } \\
\text { - Will log in to the application with username and password; } \\
\text { - He will be redirected to the map environment after authentication; } \\
\text { - Will have the possibility to use map navigation (zoom, pan); } \\
\text { - Will have the possibility to register the coordinates when collecting information in the field with some main attributes. }\end{array}$ \\
\hline Viewer & $\begin{array}{l}\text { Viewer is every public user that opens the WebGIS portal through a browser. } \\
\text { - User does not need authentication, so he will have direct access to the map environment; } \\
\text { - The user will have the possibility to use map navigation (zoom, pan), measure function (distance, area), get info, } \\
\text { printing, search and query. }\end{array}$ \\
\hline
\end{tabular}

\section{Application and database security}

In a web-based application security is a very important element. For this reason, the application security will be in different levels:

- Application level - where the user authentication will be processed through the https protocol and the use of map services will be achieved through a proxy that will enable high security in data storage;
- Database level - where sensitive data regarding user passwords will be stored encrypted in order to avoid the possibility of reading them. Also users will be stored in a separate table and the database credentials will be distributed only to the DB (IT) Administrator.

\section{Concept of database structure}

In the Figure 4 is presented a concept for database structure: 


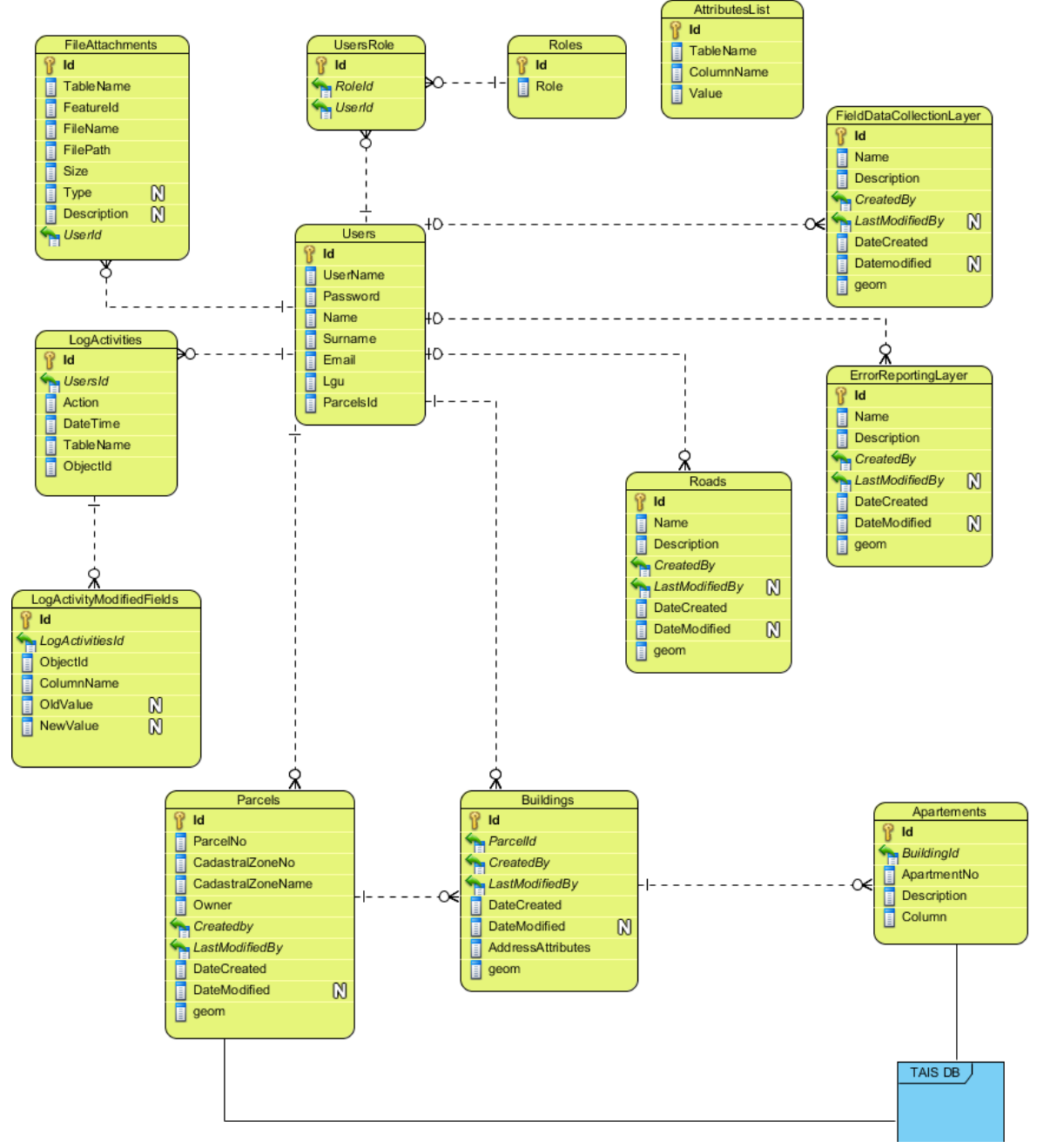

Figure 4: Conceptual WebGIS DB structure

The WebGIS application will use the data saved in a database application. We will use PostgreSQL/PostGIS as Database Engine. The WebGIS application will feature automatic backup procedures for database information.

\section{Conclusions}

As we mentioned in our article, creating a custom WebGIS application enables the possibility to create different analysis and to manage geospatial information. Tax management through WebGIS systems can help Local government units to have a more reliable cost-effective solution in local governance. In this article we have defined the possibility to create and manage the geospatial information through web Gis services. Creating custom web Gis systems gives the possibility to the users to manage all the processes into one single application with very little resources as only the web browser and internet connection. Giving the possibility to create and register data in a structured way through spatial databases, opens a lot of possibilities in the analyzing process of these data.Registering the information in a standardized way makes it easy for managing and analyzing.
[2] Wiley, Edited by John Stillwell \& Graham Clarke (2004). "Applied GIS and Spatial Analysis."

[3] Ian Gilfoyle \& Peter Thorpe (2004). "Geographical Information Management in Local Government."

[4] Philippe Rigaux, Michel Scholl \&Agnes Voisard (2002). "Spatial Databases, With Application to GIS."

\section{References}

[1] B.Drishti (2012).“GIS nëplanifikimin e zhvillimittëqëndrueshëmtënjërajoni”. 